\title{
LIVROS ESCRITOS E COLETÂNEAS ORGANIZADAS POR ENI DE MESQUITA SAMARA $(1948-2011)$
}

\section{Livros}

SAMARA, Eni de Mesquita. A família brasileira. São Paulo: Brasiliense, 1983.

SAMARA, Eni de Mesquita; COSTA, Iraci del Nero da. Demografia histórica: bibliografia brasileira. São Paulo: IPE-USP; FINEP, 1984.

SAMARA, Eni de Mesquita. As mulheres, o poder e a família. São Paulo: Anpuh; Marco Zero; Fapesp, 1989.

SAMARA, Eni de Mesquita; SOIHET, Rachel; MATOS, Maria Izilda S. de. Gênero em debate: trajetória e perspectivas na historiografia contemporânea. São Paulo: EDUC, 1997.

SAMARA, Eni de Mesquita. A colônia na bibliografia recente (1970-1998). São Paulo: Humanitas; CEDHAL, 1999.

SAMARA, Eni de Mesquita. Família e vida doméstica no Brasil: do engenho aos cafezais. São Paulo: Humanitas, 1999.

NOZOE, Nelson; BASSANEZI, Maria Silvia C. Beozzo; SAMARA, Eni de Mesquita. Os refugiados da seca: emigrantes cearenses, 1888-1889. São Paulo; Campinas: NEHD; NEPO; CEDHAL, 2003.

SAMARA, Eni de Mesquita. Família, mulheres e povoamento: São Paulo, século XVII. Bauru: Edusc, 2003.

SAMARA, Eni de Mesquita. Lavoura canavieira, trabalho livre e cotidiano: Itu, 1780-1830. São Paulo: Edusp, 2005.

SAMARA, Eni de Mesquita; DIAS, Madalena Marques; BIVAR, Vanessa dos Santos Bodstein. Paleografia e fontes do período colonial brasileiro. São Paulo: Humanitas; FFLCH; USP, 2005.

SAMARA, Eni de Mesquita; TUPY, Ismênia Silveira. História \& Documento e metodologia de pesquisa. Belo Horizonte: Autêntica, 2007. 


\section{Coletâneas}

BRESCIANI, Maria Stella; SAMARA, Eni de Mesquita; LEWKOWICZ, Ida (Orgs.). Jogos da política: Imagens, representações e práticas. São Paulo: Anpuh; Marco Zero, 1992.

SAMARA, Eni de Mesquita (Org.). Raça e família no Brasil. São Paulo: Humanitas, 1997.

SAMARA, Eni de Mesquita (Org.). As idéias e os números do gênero: Argentina, Brasil e Chile no século XIX. São Paulo: Hucitec; Cedhal; Fundação Vitae, 1997.

SAMARA, Eni de Mesquita (Org.). Catálogo bibliográfico: Trabalho feminino, cidadania e globalização. São Paulo: Humanitas, 1997.

SAMARA, Eni de Mesquita (Org.). Trabalho feminino e cidadania. São Paulo: Humanitas, 2000.

SAMARA, Eni de Mesquita (Org.). Racismo \& racistas: Trajetória do pensamento racista no Brasil. São Paulo: Humanitas, 2001.

SAMARA, Eni de Mesquita (Org.). Historiografia brasileira em debate: Olhares, recortes e tendências. São Paulo: Humanitas, 2002.

CHACÓN JIMÉNEZ, Francisco; IRIGOYEN LÓPEZ, Antonio; SAMARA, Eni de Mesquita; LOZANO ARMENDARES, Teresa (Orgs.). Sin distancias: Familia y tendencias historiográficas en el siglo XX. Murcia: Universidad de Murcia; Universidad Externado de Colombia, 2003.

SAMARA, Eni de Mesquita (Org.). Diretores da Escola Politécnica da Universidade de São Paulo: Vidas dedicadas a uma instituição. São Paulo: Escola Politécnica da Universidade de São Paulo, 2003.

SAMARA, Eni de Mesquita; FACCIOTTI, Maria Cândida Reginato (Orgs.). Mulheres politécnicas: Histórias e perfis. São Paulo: Escola Politécnica da USP, 2004.

SAMARA, Eni de Mesquita (Org.). Populações: (Con)vivência e (in)tolerância. São Paulo: Humanitas, 2004.

SAMARA, Eni de Mesquita (Org.). 30 Anos de Fuvest: A história do vestibular da Universidade de São Paulo, 1976-2006. São Paulo: Edusp, 2007.

SAMARA, Eni de Mesquita (Org.). Paleografia, documentação e metodologia histórica. São Paulo: Humanitas, 2010.

SAMARA, Eni de Mesquita (Org.). Mulheres na América e no mundo ibérico. São Paulo: Humanitas, 2012. 